\title{
Einfluss von Vorschädigung auf das Reibungsverhalten nasslaufender Lamellenkupplungen im Dauerschaltbetrieb
}

\author{
Thomas Schneider ${ }^{1}$ (D) $\cdot$ Katharina Völkel $^{1}$ (D) $\cdot$ Hermann Pflaum $^{1}$ (D) $\cdot$ Karsten Stahl $^{1}$ (D) \\ Eingegangen: 4. Januar 2021 / Angenommen: 7. September 2021 / Online publiziert: 23. November 2021 \\ (c) Der/die Autor(en) 2021
}

\section{Zusammenfassung}

Ausfälle von Lamellenkupplungen müssen aufgrund von sicherheitskritischen Funktionalitäten im Antriebsstrang sicher ausgeschlossen werden. Neben Langzeitveränderungen bei Lamellenkupplungen sind insbesondere Spontanschäden von Relevanz. Dieser Beitrag befasst sich mit dem Einfluss von Spontanschäden auf das Schaltverhalten im Dauerschaltbetrieb. Zunächst werden gezielt Spontanschäden durch Schaltungen mit hohen Belastungen hervorgerufen. Dazu werden drei Kupplungen in Stufenversuchen mit unterschiedlichen Intensitäten vorgeschädigt, so dass Spontanschäden (lokale Verfärbung, lokale Verdickungen) auftreten. Im zweiten Schritt werden Dauerschaltversuche mit den vorgeschädigten Kupplungspaketen und einer nicht vorgeschädigten Referenzkupplung durchgeführt. Das Reibungsverhalten der Kupplungen während der Dauerschaltversuche wird verglichen und bewertet. Lokale Verfärbungen sind nach dem Dauerschaltversuch in abgeschwächter Form sichtbar. An den Stahllamellen sind weiterhin lokale Verdickungen vorhanden. Im Dauerschaltversuch ist die mittlere Reibungszahl $\mu_{\text {mit }}$ der vorgeschädigten Kupplungspakete tendenziell höher. Darüber hinaus sind drehzahlabhängige Drehmomentschwankung zu beobachten Die Schwankungen sind umso stärker, je stärker die Vorschädigung war.

\section{Influence of Pre-damage on the Friction Behaviour of Wet-running Multi-plate Clutches in an Endurance Test}

\begin{abstract}
Breakdowns of multi-disc clutches must be avoided because of safety-critical functionalities in powertrains. Besides long-term damage of multi-plate clutches, spontaneous damage is of major relevance. This paper is about the influence of spontaneous damage on the shifting behavior during continuous shifting operation. First, spontaneous damage is caused by shifting operations with high loads. Therefore, three clutches are pre-damaged in step tests with different load levels to cause spontaneous damage (local discoloration, local thickening). In a second step, continuous shifting tests are performed with the three pre-damaged clutch packs and an undamaged reference clutch. During the continuous shifting tests, the friction behavior of the clutches is measured and compared. Local discolorations are visible in a reduced form after the continuous shifting tests. Local thickenings are still present on the steel plates. In the continuous shifting test, the average coefficient of friction $\mu_{\text {mit }}$ with the pre-damaged clutch packs tends to be higher. In the continuous shifting test, speed-dependent fluctuations of the friction torque can be observed. The stronger the previous damage was, the greater the fluctuations were.
\end{abstract}

Availability of data and material Die Verfügbarkeit der Daten, die für die vorliegende Studie unter Lizenz verwendet wurden, ist eingeschränkt und daher nicht öffentlich zugänglich. Auf Anfrage und mit Genehmigung der FVA sind die Daten bei den Autoren erhältlich.

\footnotetext{
Thomas Schneider

schneider@fzg.mw.tum.de
}

\author{
Forschungsstelle für Zahnräder und Getriebebau \\ (FZG), Technische Universität München, \\ Boltzmannstr. 15, 85748 Garching b. München, Deutschland
}




\section{Einleitung}

Nasslaufende Lamellenkupplungen und -bremsen sind in der Antriebstechnik häufig eingesetzte Maschinenelemente. Sie sind unter Differenzdrehzahl schaltbar und das übertragbare Drehmoment kann während des Betriebs flexibel einstellt werden. Daraus ergeben sich vielfältige Einsatzmöglichkeiten, zum Beispiel als Anfahr- und Lastschaltelemente in Doppelkupplungsgetrieben, Wandlerüberbrückungskupplungen, Differenzialsperren, Bremsen in Baumaschinen, Wendeelemente in Schiffsgetrieben oder Rutschkupplungen.

Aufgrund der oft sicherheitskritischen Funktionen, die Lamellenkupplungen im Antriebsstrang erfüllen, müssen Ausfälle sicher ausgeschlossen werden. Spontanschäden sind insbesondere problematisch, da sie durch eine einzige Schaltung zum Versagen der Kupplung führen können und daher nicht im Voraus wahrzunehmen sind.

Die Auslegung von Lamellenkupplungen im Hinblick auf Spontanschäden wird durch die physikalischen und tribologischen Eigenschaften der diversen Reibwerkstofftypen (organisch und metallisch) und Öle erschwert. In diesem Beitrag wird der Einfluss der Spontanschädigung auf das Schaltverhalten von Kupplungen mit Papierreibbelag im Dauerschaltversuch untersucht.

Spontanschäden treten aufgrund von Überlastung innerhalb weniger Schaltungen auf. $\mathrm{Zu}$ den bekanntesten Spontanschäden gehören Hot Spots für organische Reibbeläge und Sinterübertrag bei sintermetallischen Reibmaterialien. Beide Schadensformen sind unter anderem auf hohe lokale thermische Belastungen zurückzuführen.

Anderson und Knapp [1] beschreiben die Charakteristika von Hot Spots für Reibsysteme in Automobilanwendungen. Es werden lokale Hot Spots beschrieben, die elliptische bis kreisförmige Schäden aufweisen. Diese lokalen Hot Spots werden in kritische und unkritische lokale Hot Spots unterteilt. Auf den Oberflächen der unkritischen lokalen Hot Spots können nur „kosmetische“ Grenzflächenveränderungen wie z.B. Verfärbungen [2] nachgewiesen werden. An den kritischen Hot Spots treten plastische Verformungen der Stahlreibflächen auf.

Diese Beobachtungen wurden in späteren Arbeiten bestätigt [3-11]. An den kritischen Hot Spots treten sehr hohe Oberflächentemperaturen [12] auf, was zu Gefügeumwandlungen (Martensitbildung) führt $[1,2,5,12-16]$. Durch Martensitbildung kommt es zu einer Volumenzunahme und zur lokalen Verdickung der Stahllamellen [1, 16, 17]. Bei Hot Spots an Bremsscheiben werden des Öfteren hohe Spannungen und Risse beschrieben, welche aufgrund hoher thermischer Gradienten entstehen [12, 18-21].

Barber [22] benutzte die Theorie der thermoelastischen Instabilität (TEI), um die Bildung von Hot Spots zu erklären. Eine ungleiche Pressungsverteilung in der Kontakt- fläche führt zu übermäßigem Wärmeeintrag und damit zu lokalen Temperaturerhöhungen. Dies führt zu unterschiedlich starken thermischen Dehnungen, die die ursprünglich lokale unregelmäßige Pressungsverteilung verstärkt. Dieser Effekt ist selbstverstärkend und kann das Reibungssystem innerhalb weniger Schaltvorgänge zerstören. Ein umfassendes Modell zur Analyse der thermoelastischen Instabilität in nasslaufenden Lamellenkupplungen wird in [23] vorgestellt. Die Analyse zeigt den starken Einfluss des E-Moduls auf die Bildung von Hot Spots. Diese Ergebnisse wurden in experimentellen Versuchen von Fairbank [7] bestätigt.

Zur Vermeidung von Hot Spots schlägt Fieldhouse [13] aufgrund der immer vorhandenen Dickenunterschiede der Stahllamelle die Verwendung von Reibmaterialien mit einem niedrigen Elastizitätsmodul vor, so dass die Pressungsunterschiede ausgeglichen werden können. Zagrodzkis [24] Simulationsergebnisse zeigen, dass eine Verringerung der Dicke der Stahlscheibe die thermoelastische Stabilität deutlich verbessern kann. Diese konstruktive Änderung muss jedoch mit Vorsicht angewendet werden. Dünnere Stahllamellen sind anfälliger für plastische Verformungen wie z.B. Tellern. Zudem dienen die Stahllamellen als Wärmespeicher. Eine Verringerung ihrer Dicke führt zu einer Erhöhung der mittleren Temperatur. In der Literatur wird auBerdem vorgeschlagen, die Wärmeleitfähigkeit [25-27] der verwendeten Bauteile zu erhöhen [28].

Im Gegensatz zu Spontanschäden entstehen Langzeitschäden im Laufe mehrere tausend Schaltungen. Typische Schädigungsformen sind Verschleiß oder eine Veränderung des Reibungsverhaltens. Acuner et al. [29] sowie Stockinger et al. [30] führten Dauerschaltversuche mit Synchronisierungen durch und beschreiben ein Auffüllen der Porosität des Belages bei hoher thermischer Belastung für organische Kohlenstoffreibbeläge. Diese Glättung des Reibbelags durch Öl-Crackprodukte führt zu einer Verringerung des Gleitreibungskoeffizienten während des Schaltvorgangs. Die experimentellen Untersuchungen von Matsumoto [31] und Nyman et al. [32] zeigen ebenfalls einen Zusammenhang zwischen Belagporosität, Reibwert und Lebensdauer für nasslaufende Lamellenkupplungen. So wird gezeigt, dass ein poröser Belagwerkstoff in Bezug auf die thermischen Eigenschaften widerstandsfähiger ist.

Neben der Porosität hat auch die Reibflächentemperatur einen wesentlichen Einfluss auf das Lebensdauerverhalten von Kupplungen [33]. Nach Li et al. [34] können anhaltend hohe Temperaturen die Zellulosefasern des Papierreibmaterials zersetzen (Verkokung). Dadurch wird die Festigkeit des Belages verringert und die Verschleißrate potenziell erhöht. Hensel [35] berichtet über Referenzversuche bestehend aus Stufentests und Langzeittests zur Bewertung der Reibbeläge in Bezug auf spontane und kumulative Schädigung. Der Einfluss der Betriebsweise auf die Schädigung kann demnach durch Temperaturmessungen anhand 
der Einflüsse auf die Wärmebelastung der Kupplung bzw. Bremse ermittelt werden. Aus diesen Messungen werden charakteristische Werte für die Bewertung der thermischen Belastung abgeleitet, die eine Korrelation mit der Langzeitschädigung erlauben [28].

Diese Veröffentlichung erweitert den Stand der Technik, indem der Einfluss von Spontanschäden auf das Reibungsverhalten im Dauerschaltbetrieb in experimentellen Versuchen aufgezeigt wird. Im erstem Schritt werden gezielt Spontanschäden durch Schaltungen mit hohen Belastungen hervorgerufen. Dazu werden drei Kupplungen in Stufenversuchen mit unterschiedlichen Intensitäten vorgeschädigt, so dass Spontanschäden (lokale Verfärbung, lokale Verdickungen) auftreten. Im zweiten Schritt werden Dauerschaltversuche mit den vorgeschädigten Kupplungspaketen und einer nicht vorgeschädigten Referenzkupplung durchgeführt. Das Reibungsverhalten der vier Kupplungen soll im Anschluss miteinander verglichen werden.

\section{Versuchsteile und Forschungsmethode}

Die Versuche werden mit Papierreibbelag durchgeführt. Die Stahllamellen sind als Außen- und die Belaglamellen als Innenlamelle ausgeführt. Abb. 1a zeigt eine Belaglamelle sowie Abb. 1b die dazugehörige Stahllamelle; die geometrischen Größen sind in Tab. 1 aufgeführt.

Die Versuche zur Bestimmung von Reibarbeit, Rutschzeit und Belastungsgrenzen wurden auf dem Komponentenprüfstand ZF/FZG KLP-260 durchgeführt. Abb. 2 zeigt eine Skizze des Prüfstandes (nach Meingaßner et al. [36]).

Für die Versuche wird im Komponentenprüfstand ZF/ FZG KLP-260 ein vollständiges Lamellenpaket über entsprechende Mitnehmer verbaut. Der Außenmitnehmer ist fest mit dem Gehäuse verbunden. Der Innenmitnehmer ist an der Zentralwelle befestigt, die mit den beiden Schwungscheiben (J1, J2) verbunden ist. Das Reibmoment wird am Außenmitnehmer gemessen. Vor Beginn
Tab. 1 Geometrische Größen der Versuchslamellen

\begin{tabular}{ll}
\hline Reibflächeninnendurchmesser & $141 \mathrm{~mm}$ \\
Reibflächenaußendurchmesser & $168 \mathrm{~mm}$ \\
\hline
\end{tabular}

Tab. 2 Technische Daten des Prüfstands ZF/KLP-260. (Nach Meingaßner et al. [36])

\begin{tabular}{ll}
\hline $\begin{array}{l}\text { Massenträgheitsmoment } \\
\text { Flanschverschraubung }\end{array}$ & $\mathrm{J}_{1}=0,1 \ldots 0,75 \mathrm{kgm}^{2}$ \\
$\begin{array}{l}\text { Massenträgheitsmoment Kupp- } \\
\text { lung }\end{array}$ & $\mathrm{J}_{2}=1,0 \mathrm{kgm}^{2}$ \\
Lamellendurchmesser & $\mathrm{d}=75 \ldots 260 \mathrm{~mm}$ \\
Max. Reibmoment & $\mathrm{T}_{\mathrm{R}, \max }=2000 \mathrm{Nm}$ \\
Differenzdrehzahl & $\Delta \mathrm{n}=0 \ldots 7000 \mathrm{~min}^{-1}$ \\
Schlupfmoment nom./bei max & $\Delta \mathrm{n}=90 / 140 \mathrm{~min}^{-1}$ \\
Schlupfmoment nom./bei max. & $\mathrm{T}_{\text {Schlupf }}=2000 \mathrm{Nm} / 1300 \mathrm{Nm}$ \\
Schlupfdrehzahl & \\
\hline
\end{tabular}

jedes Schaltvorganges wird die Zentralwelle durch den Hauptantrieb auf die Differenzdrehzahl $\Delta \mathrm{n}$ beschleunigt. Unmittelbar vor der Schaltung wird der Hauptantrieb durch eine elektromagnetische Kupplung von der Zentralwelle getrennt. Die Aktuierung der Kupplung erfolgt hydraulisch auf die Außenlamellen. Die verschiedenen einstellbaren Parameter (Massenträgheit, Differenzdrehzahl, Pressung) bieten eine große Flexibilität bei der Versuchsplanung [28, 36].

Die technischen Daten des Prüfstands sind in Tab. 2 aufgeführt.

Nach der Beschreibung der Versuchsteile und des Prüfstands wird im folgenden Abschnitt der Versuchsablauf erläutert. Zunächst werden die Kupplungen einem Einlauf unterzogen, sodass Fertigungstoleranzen und Inhomogenitäten ausgeglichen werden. Die spezifischen Einlaufbedingungen, die sich auf die Reibfläche beziehen, sind in Tab. 3 angegeben und orientieren sich an den Werten von Völkel [37, 38].

Zur Untersuchung des Reibungsverhaltens vorgeschädigter Lamellenkupplungen im Dauerschaltbetrieb werden
Abb. 1 Versuchsteile - a Belaglamelle, b Stahllamelle

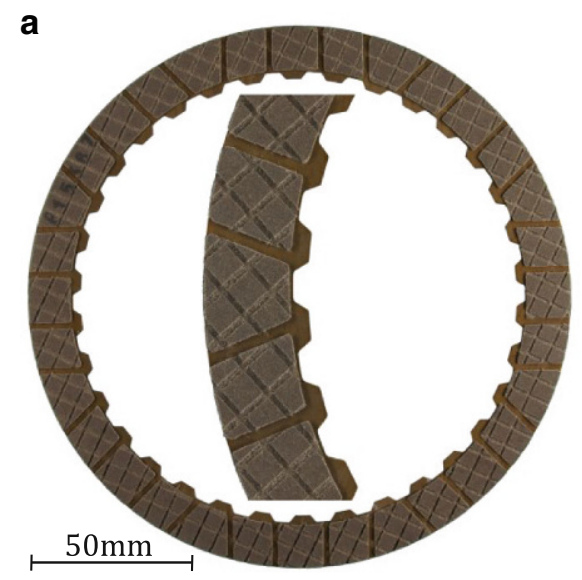

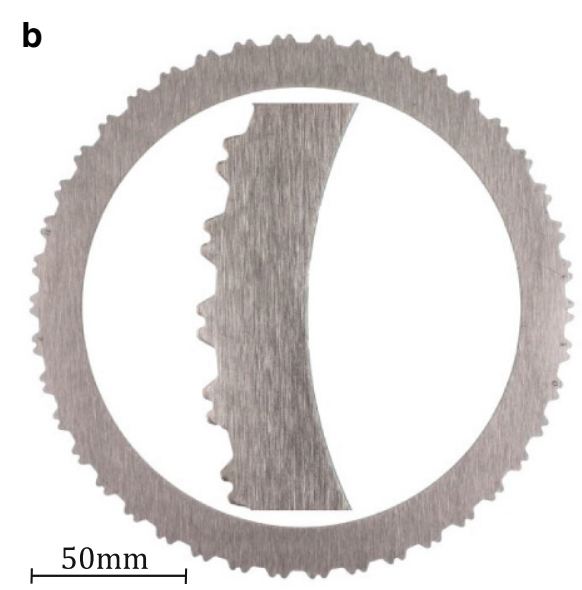


Abb. 2 Komponentenprüfstand ZF/FZG KLP-260 - Schema. (Nach Meingaßner et al. [36])

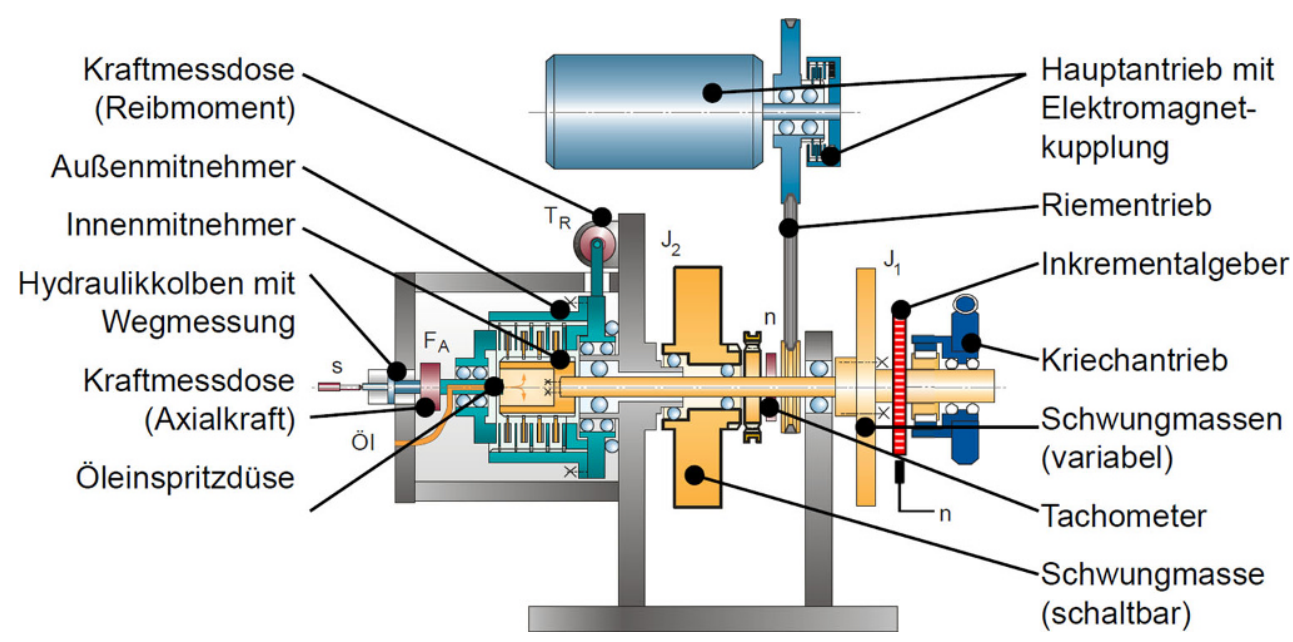

Tab. 3 Belastungen im Einlauf. (Nach Völkel $[37,38]$ )

\begin{tabular}{lllll}
\hline Einlaufstufe & Schaltungen & $\begin{array}{l}\text { Pressung } \\
\text { p in N/mm }\end{array}$ & Gleitgeschwindigkeit $\mathrm{vg}$ in $\mathrm{m} / \mathrm{s}$ & $\begin{array}{l}\text { Spez. Reibarbeit } \\
\mathrm{q} \mathrm{in} / \mathrm{mm}^{2}\end{array}$ \\
\hline 1 & 100 & 0,5 & 6,5 & 0,08 \\
2 & 200 & 1,0 & 8,0 & 0,12 \\
3 & 300 & 1,5 & 15,4 & 0,42 \\
\hline
\end{tabular}

Tab. 4 Belastungen im Stufenversuch

\begin{tabular}{lllll}
\hline Laststufe & $\begin{array}{l}\text { Spez. Reibarbeit } \\
\text { in J/mm }\end{array}$ & $\begin{array}{l}\text { Spez. Reibleistung } \\
\text { in W/mm }\end{array}$ & $\begin{array}{l}\text { Pressung } \\
\text { in N/mm }\end{array}$ & $\begin{array}{l}\text { Gleitgeschwindigkeit } \\
\text { in m/s }\end{array}$ \\
\hline 1 & 0,53 & 1,18 & 0,75 & 12,1 \\
2 & 0,76 & 1,42 & 0,75 & 14,6 \\
3 & 1,03 & 1,66 & 0,75 & 17,0 \\
4 & 1,35 & 1,89 & 0,75 & 19,4 \\
5 & 1,70 & 2,13 & 0,75 & 21,8 \\
6 & 2,10 & 2,37 & 0,75 & 24,3 \\
7 & 2,39 & 2,52 & 0,75 & 25,9 \\
8 & 2,78 & 2,72 & 0,75 & 27,9 \\
9 & 3,20 & 2,92 & 0,75 & 29,9 \\
10 & 3,55 & 3,08 & 0,75 & 31,5 \\
\hline
\end{tabular}

diese in Stufenversuchen vorgeschädigt. Die Durchführung dieser Schädigung kann als Konditionierung der Versuchsteile angesehen werden. Die Stufenversuche, die auf dem Komponentenprüfstand ZF/FZG KLP-260 im Bremsbetrieb durchgeführt werden, haben sich bereits bei Hensel [35] und Strebel et al. [39] bewährt. Im Stufentest wird die Ausgangsdrehzahl der Schaltung von Lastniveau zu Lastniveau erhöht. Dabei ist die Anzahl der Schaltungen mit zehn Schaltungen pro Lastniveau entsprechend klein, um Langzeitveränderungen der Kupplung auszuschließen, aber entsprechend groß, um stochastische Effekte bei der Entstehung von Spontanschäden zu minimieren. Nach jeder Laststufe wird die Kupplung auf Anzeichen von Schäden untersucht und alle Änderungen werden dokumentiert. Die Zykluszeit zwischen zwei Schaltungen ist lang ge- nug (40s), um die Rückkühlung des Kupplungssystems auf Öleinspritztemperatur vor jeder Schaltung zu gewährleisten. Während des Einlaufens und des Stufenversuchs beträgt der spezifische Kühlölvolumenstrom bezogen auf die Kupplungsfläche für alle Kupplungen $0,8 \mathrm{~mm}^{3} / \mathrm{mm}^{2} \mathrm{~s}$. Die Belastungsniveaus des Stufenversuchs sind in Tab. 4 aufgeführt. Alle Belastungen sind bezogen auf die Bruttoreibfläche normiert [28].

Zur besseren Übersicht werden die Belastungen im Stufenversuch auch grafisch dargestellt. Abb. 3 veranschaulicht die spezifischen Belastungsstufen eines Stufentests mit zehn Laststufen. Jeder Punkt stellt eine Laststufe des Stufentests dar.

Es werden drei Kupplungspakete im Stufenversuch unterschiedlich stark vorgeschädigt (LS-4, LS-6, LS-7). Die 
Abb. 3 Belastungen im Stufenversuch
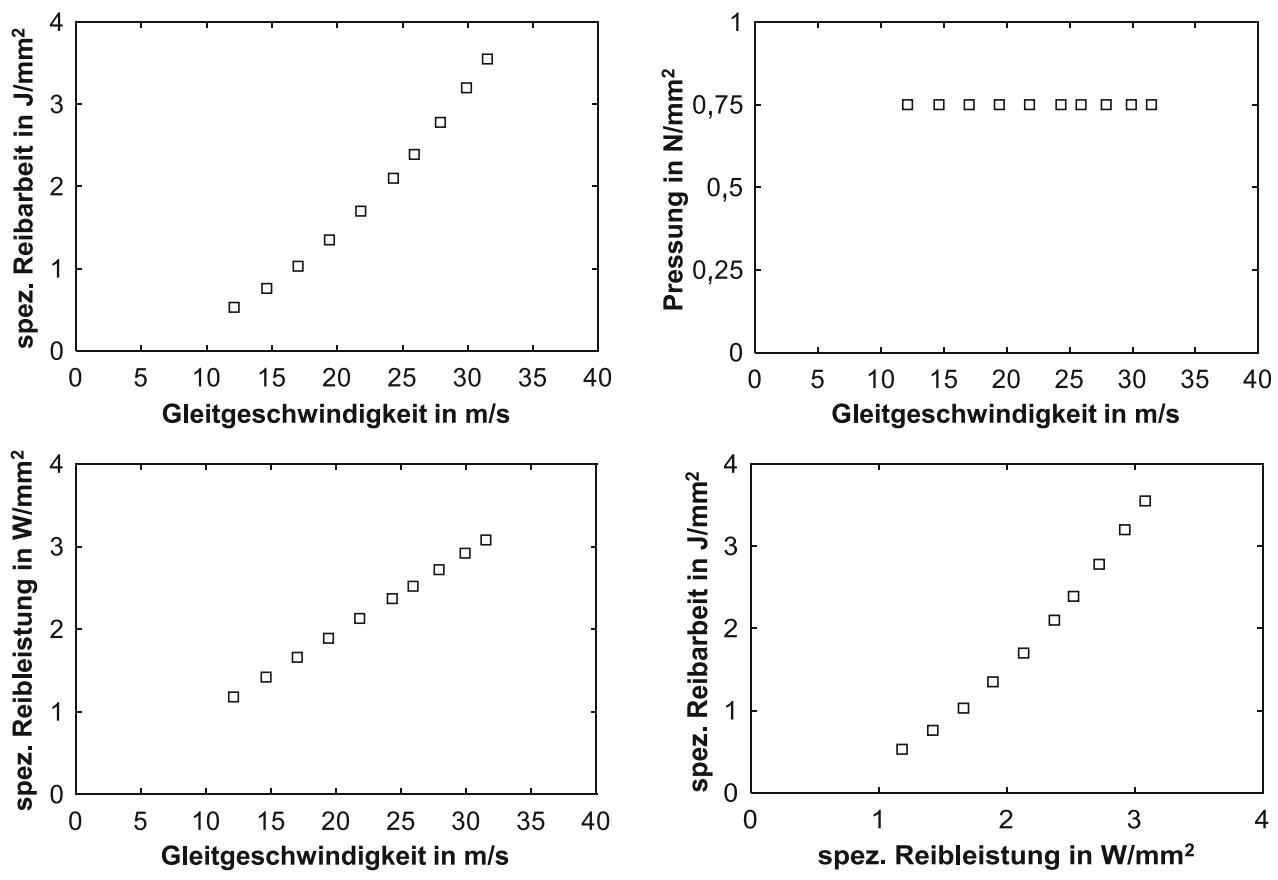

Tab. 5 Versuchsübersicht

\begin{tabular}{ll}
\hline Versuchsbezeichnung & Laststufe im Stufenversuch \\
\hline LS-0 & Kein Stufenversuch \\
LS-4 & 4 \\
LS-6 & 6 \\
LS-7 & 7 \\
\hline
\end{tabular}

Tab. 6 Belastungen im Dauerschaltversuch

\begin{tabular}{llll}
\hline Schaltungen & $\begin{array}{l}\text { Pressung } \\
\mathrm{p} / \mathrm{N} / \mathrm{mm}^{2}\end{array}$ & $\begin{array}{l}\text { Gleitgeschwin- } \\
\text { digkeit } \\
\mathrm{vg} / \mathrm{m} / \mathrm{s}\end{array}$ & $\begin{array}{l}\text { Spez. Reibarbeit } \\
\mathrm{q} / \mathrm{J} / \mathrm{mm}^{2}\end{array}$ \\
\hline 10.000 & 1,0 & 13,8 & 0,73 \\
\hline
\end{tabular}

Kupplungspakete des Referenzversuchs werden nicht vorgeschädigt (LS-0). Für diesen folgt nach dem Einlauf direkt der Dauerschaltversuch ohne schädigenden Stufenversuch. Tab. 5 stellt eine Versuchsübersicht dar.

Im Anschluss an den Stufenversuch folgt der Dauerschaltversuch mit Belastungen gemäß Tab. 6. Während des

Abb. 4 a Belastungen für die Vorschädigung bei Laststufe 4, b exemplarischer Reibungszahlverlauf in der höchsten Laststufe

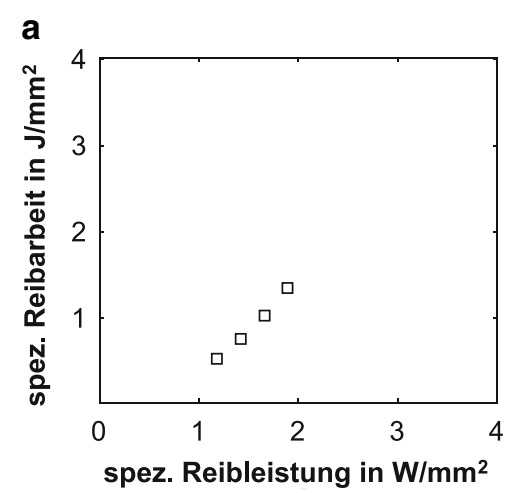

Dauerversuchs betragen der spezifische Kühlölvolumenstrom $0,8 \mathrm{~mm}^{3} / \mathrm{mm}^{2} \mathrm{~s}$ und die Zykluszeit $15 \mathrm{~s}$.

\section{Versuchsergebnisse}

Für den Versuch LS-4 wurde der Stufenversuch bis zur Laststufe 4 durchgeführt (vgl. Tab. 4, Abb. 4a). Unter diesen Belastungen kommt es zu ersten lokalen Verfärbungen auf den Stahllamellen. Diese Schädigungsform wurde in der Literatur mehrfach beschrieben [1, 6, 40]. Der Reibungszahlverlauf für eine exemplarische Schaltung auf diesem Lastniveau ist in Abb. 4b abgebildet. Neben drehzahlabhängigen Schwankungen im Reibungszahlverlauf können keine Auffälligkeiten beobachtet werden. Dem Stufentest folgte der Dauerversuch.

Abb. 5a zeigt die Stahl- und Belaglamelle direkt nach dem Stufentest. Die Stahllamellen zeigen erste lokale Verfärbungen. Diese sind nach dem Dauerschaltversuch schwä- 
Abb. 5 Fotos der vorgeschädigten Stahl- und Belaglamellen a nach Laststufe 4, b nach dem Dauerschaltversuch
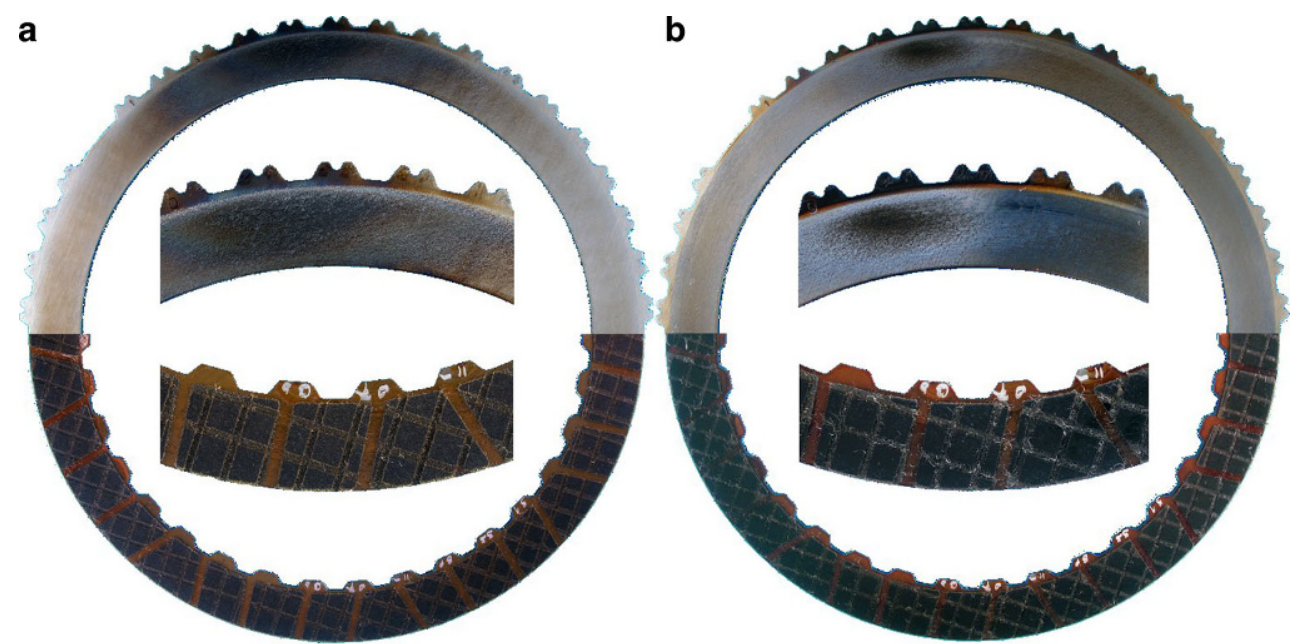

cher erkennbar (vgl. Abb. 5b). Die Belaglamellen zeigen nach dem Dauerschaltversuch geringe Verschleißspuren.

Der Stufenversuch für Versuch LS-6 wurde bis Laststufe 6 ausgeführt. Die Belastungen sind Tab. 4 sowie Abb. 6a zu entnehmen. Der Stufentest wurde so lange durchgeführt, bis starke lokale Verfärbung auf der Stahllamelle auftreten. Gleichzeitig ist ein Auffächern der drehzahlabhängigen Schwankungen im ersten Drittel der Schaltung zu beobachten (vgl. Abb. 6b).

Abb. 7 stellt die Stahl- und Belaglamellen direkt nach dem Stufentest sowie nach dem Dauerschaltversuch dar. Auf der rechten Seite in Abb. 7 sind starke lokale Verfärbungen auf der Stahllamelle erkennbar. Diese sind nach dem Dauerschaltversuch in ihrer Erscheinungsform weniger stark auffallend. Die Belaglamellen zeigen nach dem Dauerschaltversuch geringe Verschleißspuren.

Abb. 8a zeigt die Belastungen für Versuch LS-7 sowie den Reibungszahlverlauf einer Schaltung auf Laststufe 7. Neben lokalen Verfärbungen auf der Stahllamelle treten lokale Verdickungen auf. Diese Verdickungen $(<0,1 \mathrm{~mm})$ wurden mit einer Bügelmessschraube gemessen. Gleichzeitig ist ein starkes Auffächern der drehzahlabhängigen Schwankungen über die gesamte Schaltung hinweg zu beobachten. Dem Stufentest folgte der Dauerversuch.
Analog zu den vorherigen Darstellungen zeigt Abb. 9a die Stahl- und Belaglamelle direkt nach dem Stufentest. Auf der rechten Seite (Abb. 9b) ist der Zustand der Lamellen nach dem Dauerschaltversuch dokumentiert. Die Fotos zeigen, dass lokale Verfärbungen sich im Laufe des Dauerschaltversuches abschwächen. Lokale Verdickungen sind auch nach dem Dauerschaltversuch messbar vorhanden. Der Reibbelag zeigt nach dem Stufenversuch Verschleißspuren, die sich im Laufe der 10.000 Schaltungen deutlich verstärken.

Abb. 10 zeigt die Reibungszahlverläufe der letzten 10 Schaltungen im Einlauf für Versuch LS-4. Es ist ersichtlich, dass sich die Reibungszahlverläufe der letzten 10 Schaltungen nur minimal unterscheiden. Diese Erkenntnis ist für die Auswertung und Bewertung der nachfolgenden Messschriebe von Bedeutung.

Die Abb. 11, 12 und 13 zeigen die Reibungszahlverläufe der drei vorgeschädigten Kupplungen (LS-4, LS-6 und LS7) und der nicht vorgeschädigten Referenzkupplung (LS-0) für ausgewählte Schaltungen im Dauerschaltversuch.

Für Schaltung 1 im Dauerversuch (vgl. Abb. 11) sind die Reibwerte zu Beginn der Schaltung von Versuch LS-4 am höchsten. Die Werte von LS-0 und LS6 liegen auf dem gleichen Niveau. LS7 zeigt vergleichsweise geringe Rei-
Abb. 6 a Belastungen für die Vorschädigung bei Laststufe 6 , b exemplarischer Reibungszahlverlauf in der höchsten Laststufe
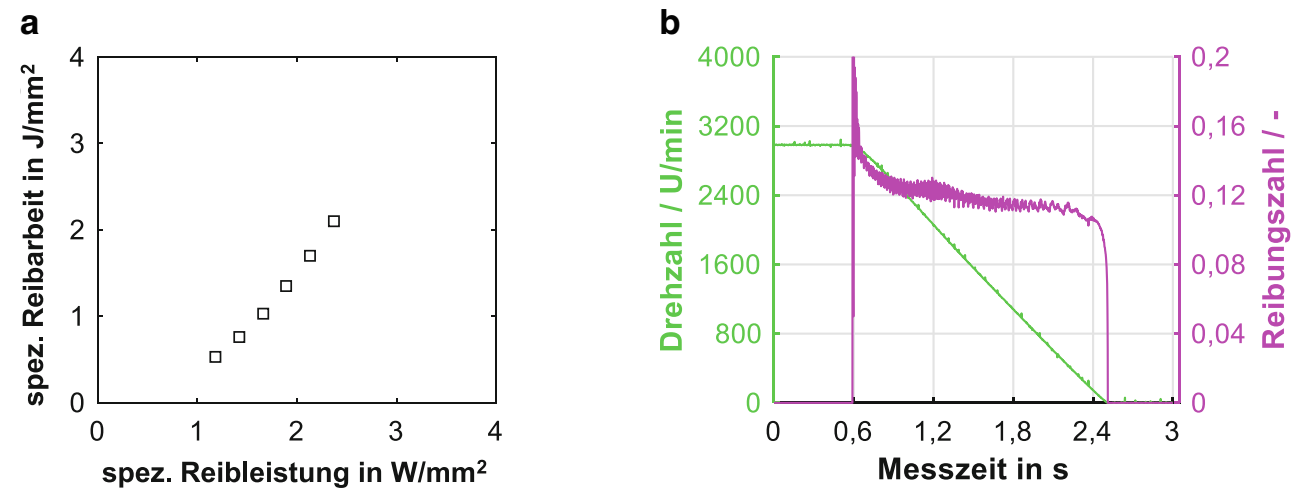
Abb. 7 Fotos der vorgeschädigten Stahl- und Belaglamellen a nach Laststufe 6, b nach dem Dauerschaltversuch
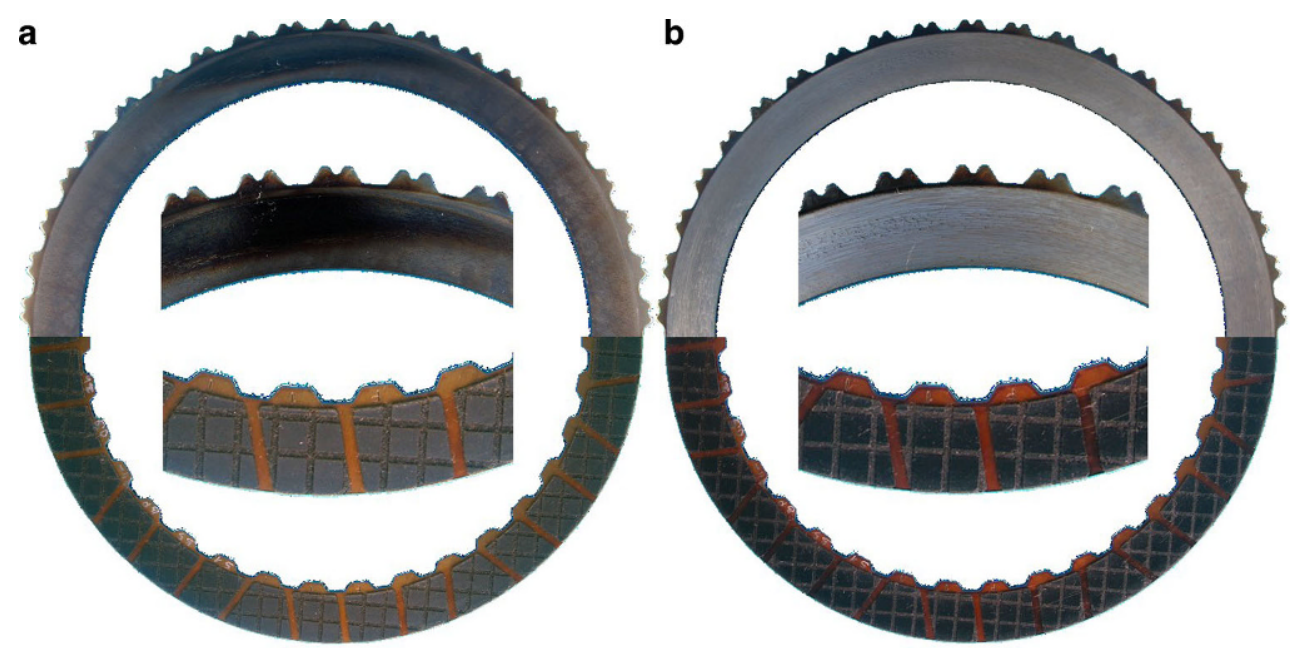

Abb. 8 a Belastungen für die Vorschädigung bei Laststufe 7 , b exemplarischer Reibungsverlauf in der höchsten Laststufe

Abb. 9 Fotos der vorgeschädigten Stahl- und Belaglamellen a nach Laststufe 7, b nach dem Dauerschaltversuch
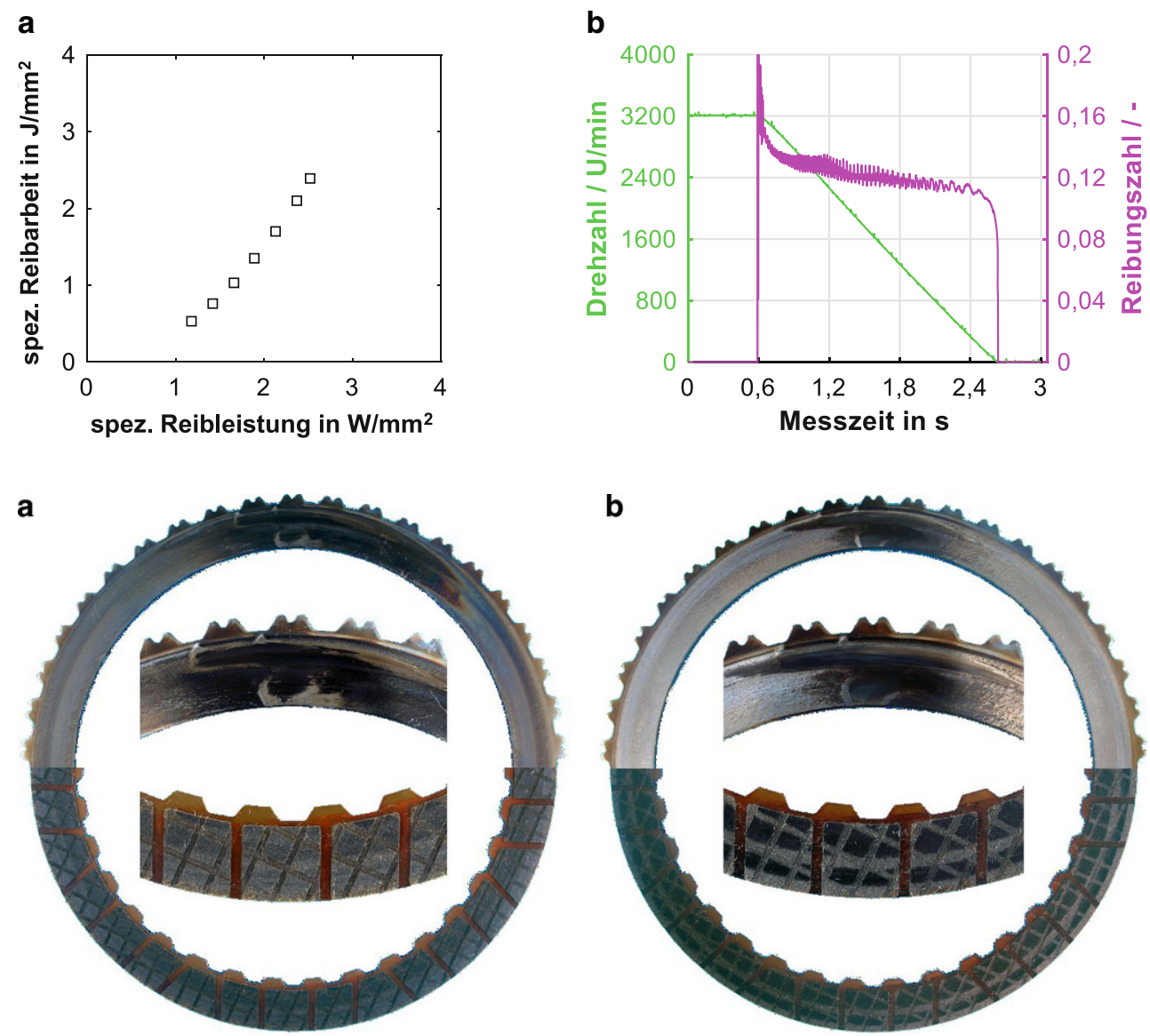

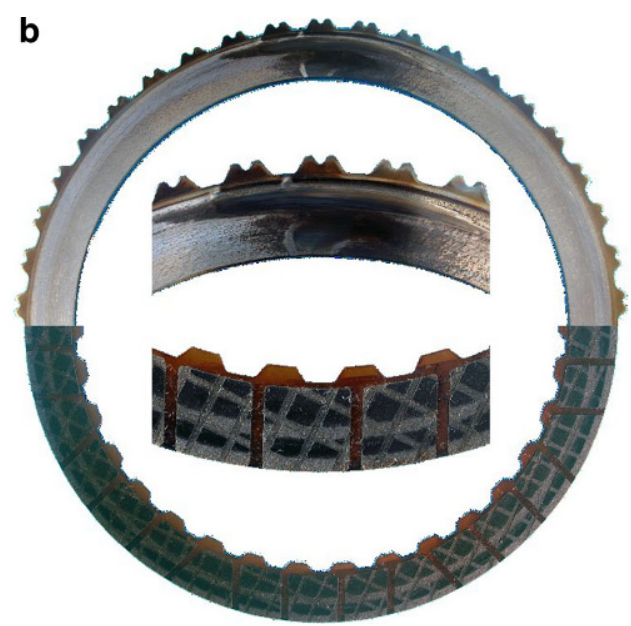

bungszahlen zu Schaltungsbeginn. Am Ende der Schaltung befinden sich die unterschiedlichen Kupplungen auf dem gleichen Reibungszahlniveau. Bei Versuch LS-7 nimmt die Reibungszahl mit Abnahme der Gleitgeschwindigkeit leicht zu, währenddessen sich das Reibungszahlniveau bei den anderen Versuchen auf dem gleichen Niveau hält. Die Messungen zeigen drehzahlabhängige Schwankungen der Reibungszahl. Umso stärker die Vorschädigungen der
Versuchsteile sind, desto stärker fallen die Schwankungen aus.

Abb. 12 zeigt die Reibungsverläufe über der Gleitgeschwindigkeit für Schaltung 5000 im Dauerschaltversuch. Das Lamellenpaket, das auf Laststufe 7 vorgeschädigt wurde, weist zu Beginn der Schaltung niedrigere Reibungszahlen als die Vergleichspakete auf. Bei Gleitgeschwindigkeiten zwischen 12 und $11 \mathrm{~m} / \mathrm{s}$ zeigen alle Versuchspakete 
Abb. 10 Reibungszahlverläufe der letzten 10 Schaltungen des Einlaufs von Versuch LS-4

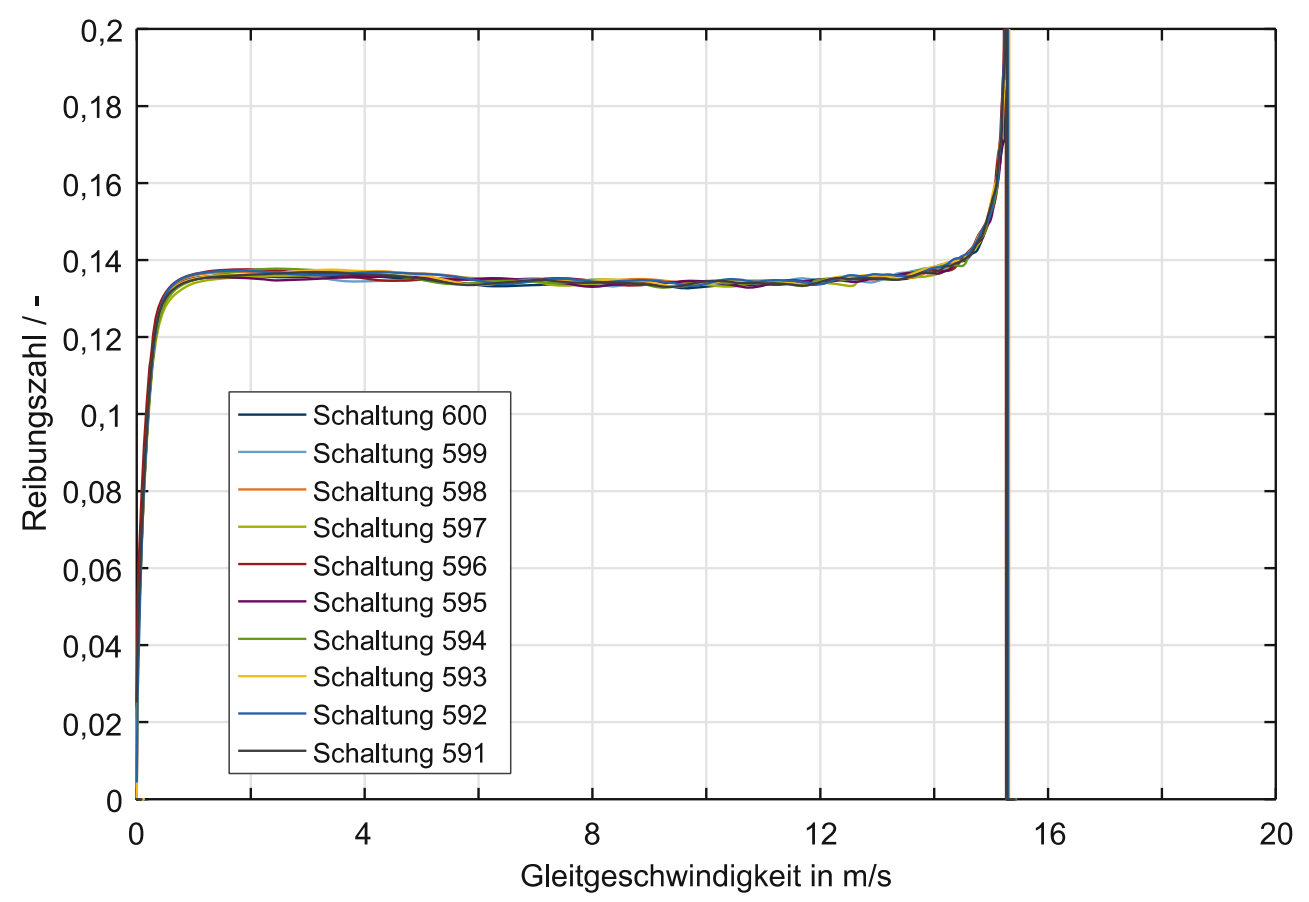

Abb. 11 Einzelschaltungsvergleich vorgeschädigter und nicht vorgeschädigter Lamellenpakete (Schaltung 10 im Dauerschaltversuch)

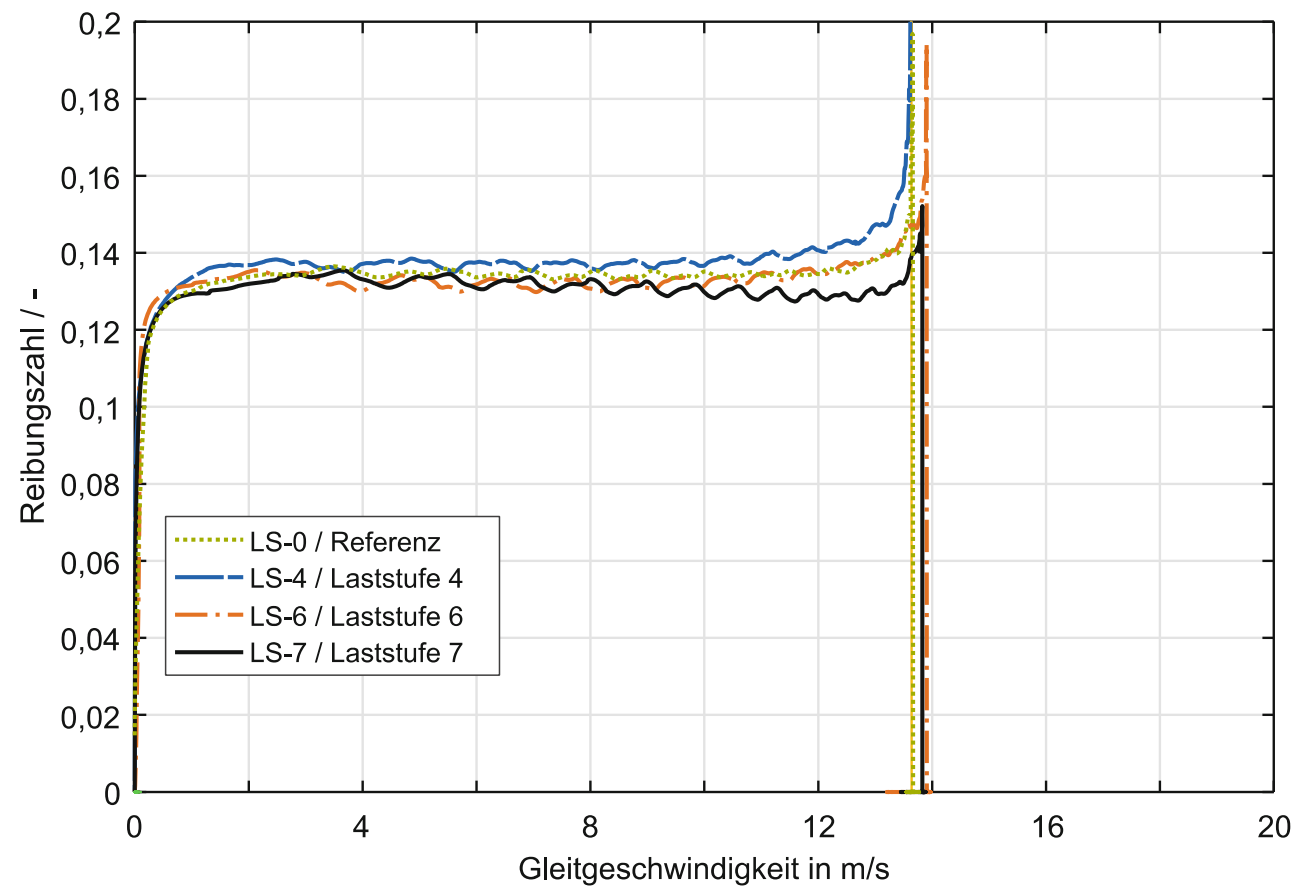

die identische Reibcharakteristik. Mit Abnahme der Gleitgeschwindigkeit kommt es zu Unterschieden zwischen den Versuchen. Der Gradient des Reibwerts über der Gleitgeschwindigkeit ist mit abnehmender Gleitgeschwindigkeit in den vorgeschädigten Kupplungspaketen größer als in der Referenzkupplung. Die Messungen zeigen drehzahlabhängige Schwankungen des Reibmoments und damit der Reibungszahl für alle Versuchspakete.
Für Schaltung 10.000 im Dauertest zeigt sich ein ähnliches Bild wie bei Schaltung 5000 (vgl. Abb. 13). Eine Zunahme des Reibungskoeffizienten bei Abnahme der Gleitgeschwindigkeit ist bei allen Kupplungspaketen zu beobachten. Diese Zunahme ist bei vorgeschädigten Versuchspaketen stärker als bei dem Referenzpaket. Zusätzlich ist bei den vorgeschädigten Systemen eine stärkere drehzahlabhängige Schwankung des Reibwertes zu beobachten. 
Abb. 12 Einzelschaltungsvergleich vorgeschädigter und nicht vorgeschädigter Lamellenpakete (Schaltung 5000 im Dauerschaltversuch)

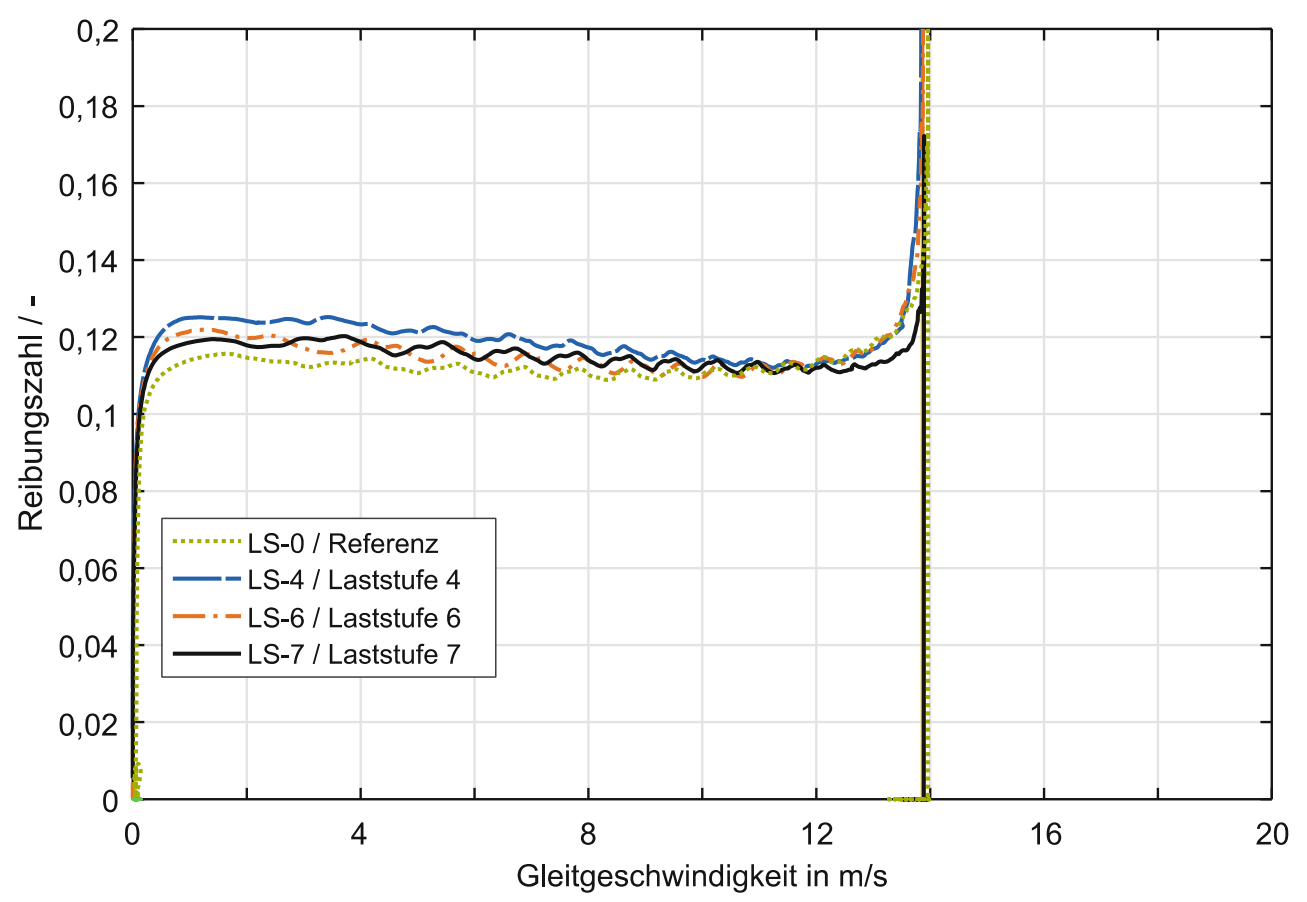

Zusätzlich zu den einzelnen Schaltungen sind in Abb. 14 die Trendkurven für die mittlere Reibungszahl $\mu_{\text {mit }}$ der vier im Dauerschaltversuch untersuchten Kupplungen dargestellt. Die mittlere Reibungszahl $\mu_{\text {mit }}$ entspricht dem arithmetischen Mittelwert der Reibungszahl im Bereich 0 bis $60 \%$ der maximalen Gleitgeschwindigkeit $\mathrm{v}_{\mathrm{g}, \max }$ [41]. Nachdem sich ein stabiles Reibungszahlniveau zu Beginn des Versuchs für alle Kupplungspakete gebildet hat, fallen alle vier Trendkurven im Laufe des Versuchs ab. Die drei vorgeschädigten Systeme befinden sich zu Ende des Versuchs auf ähnlichem Reibungszahlniveau. Beim Referenzsystem werden zu Versuchsende niedrigere Werte als bei den vorgeschädigten Systemen gemessen.
Abb. 13 Einzelschaltungsvergleich vorgeschädigter und nicht vorgeschädigter Lamellenpakete (Schaltung 10.000 im Dauerschaltversuch)

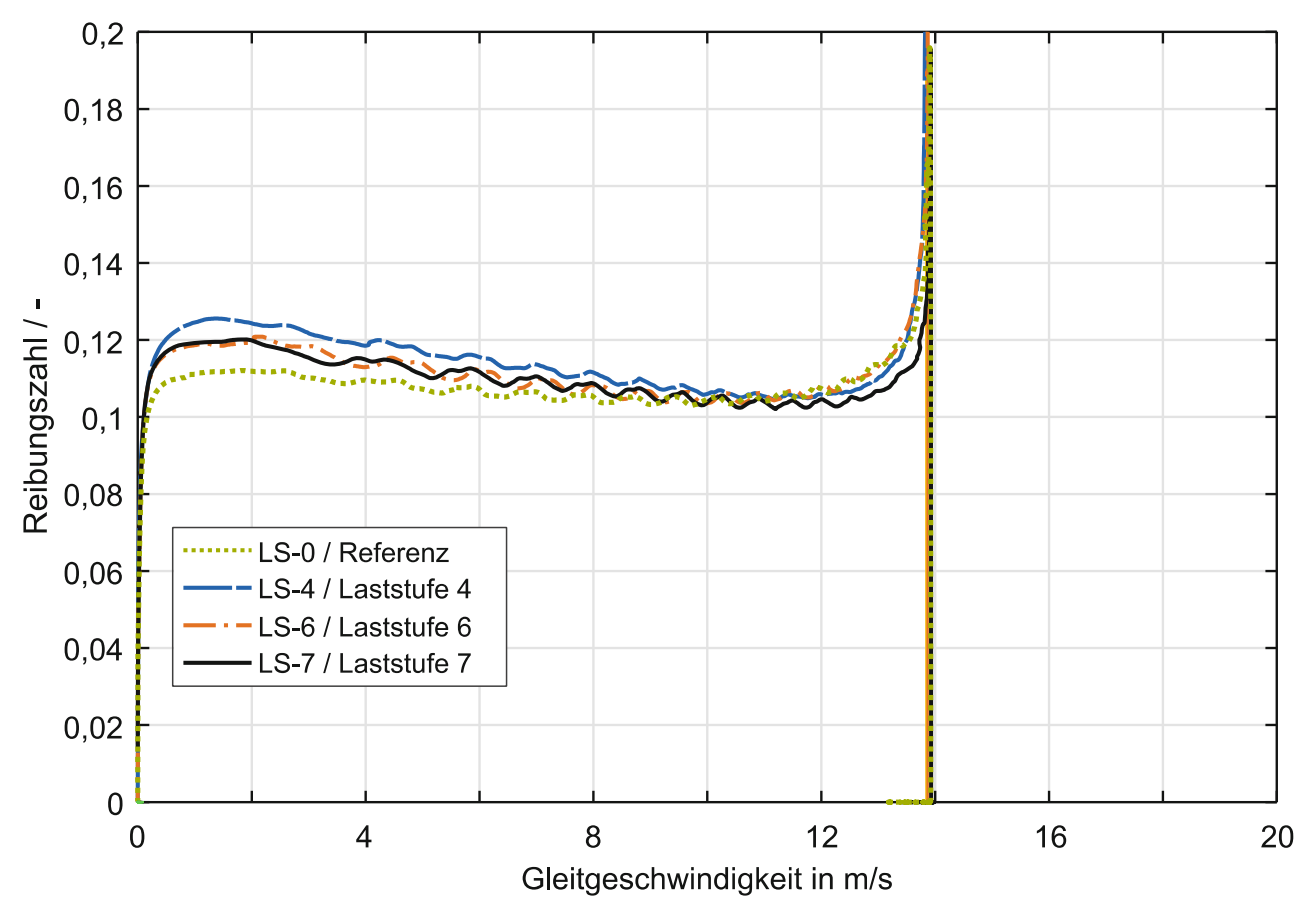


Abb. 14 Trendvergleich von $\mu$ mit über 10.000 Schaltungen im Dauerschaltversuch

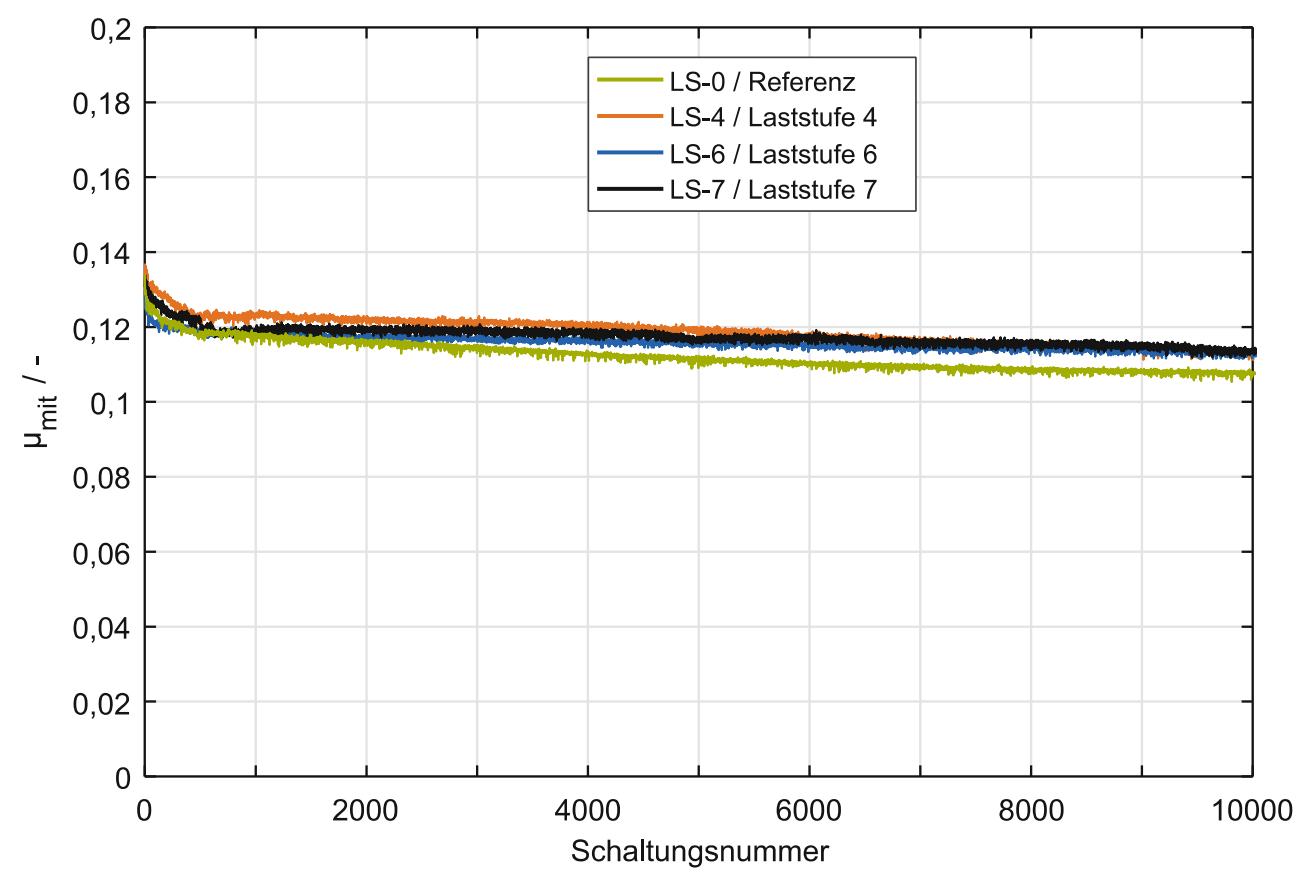

\section{Diskussion}

Im Stufentest führen die verschiedenen Lastniveaus zu unterschiedlichen Schädigungsformen. Lokale Verfärbungen und lokale Verdickungen können beobachtet werden. Nach dem Dauertest sind lokale Verfärbungen noch in abgeschwächter Form sichtbar. Lokale Verdickungen sind weiterhin auf den Stahllamellen vorhanden. Dies zeigt, dass lokale Verfärbungen, die von Duminy [42] auch als Ölcrackprodukte beschrieben werden, durch Verschleißmechanismen während des Dauerschaltversuchs wieder teilweise abgetragen werden. Es hat sich gezeigt, dass die am stärksten geschädigte Kupplung bei hohen Gleitgeschwindigkeiten, also zu Schaltungsbeginn eindeutig niedrigere Reibungszahlen aufweist als die restlichen getesteten Pakete. Von Acuner [43] wurde im Falle von Carbon-Synchronisierung dieses Verhalten damit begründet, dass der Reibbelag durch Ölablagerungen eingeglättet wurde. Es ist zu vermuten, dass dieses Phänomen auch hier eintritt und dass die Belagporen teilweise verstopft und verschmiert werden. Zusätzlich ist bei den vorgeschädigten Systemen eine stärkere drehzahlabhängige Schwankung des Reibmoments zu beobachten. Je stärker die Vorschädigungen der Versuchsteile sind, desto stärker fallen die Schwankungen aus. In nachfolgenden Untersuchungen sollen Kreuztest durchgeführt werden, sodass man die Effekte (Abfall Reibungszahl, Drehzahlschwankungen) den geschädigten Lamellen (Belag-/Stahllamelle) zuordnen kann. Die vorgeschädigten Lamellen sollen mit Lamellen im Neuzustand gepaart werden. Darüber hinaus sollen Versuche mit Temperaturmessungen durchgeführt werden, damit eine Abhängigkeit des Schädigungseintritts sowie des Ab- falls der Reibungszahl vom Temperaturniveau betrachtet werden kann.

\section{Zusammenfassung}

In dieser Veröffentlichung werden Untersuchungen des Einflusses von Spontanschäden auf das Reibungsverhalten einer Lamellenkupplung im Dauerschaltbetrieb beschrieben. Drei Kupplungen wurden im Stufenversuch bei unterschiedlichen Belastungen vorgeschädigt. Es traten leichte und starke lokale Verfärbungen, sowie lokale Verfärbungen mit lokalen Verdickungen auf. Im Anschluss folgte ein Dauerschaltversuch (10.000 Schaltungen) mit drei vorgeschädigten Kupplungen und einer nicht vorgeschädigten Referenzkupplung. Nach dem Dauerschaltversuch sind die lokalen Verfärbungen nur mehr schwächer erkennbar. Lokale Verdickungen sind weiterhin auf der Stahllamelle vorhanden. Im Dauerschaltversuch sind drehzahlabhängige Schwankungen des Reibmoments zu beobachten. Je stärker die Vorschädigung war, desto stärker waren die Schwankungen, welche auf Dickenunterschiede im Kupplungspaket zurückzuführen sind. Im Trendverlauf zeigt sich, dass die mittlere Reibungszahl $\mu_{\text {mit }}$ der vorgeschädigten Kupplungspakete auf höherem Niveau liegt als bei der Referenzkupplung. Es hat sich gezeigt, dass mit stark geschädigten Lamellen noch mehrere tausend Schaltungen durchgeführt werden können. Eine Verschlechterung des Reibungsverhaltens kann beobachtet werden.

Danksagung Die vorgestellten Erkenntnisse basieren auf dem Forschungsvorhaben FVA-Nr. 515/V; gefördert aus Eigenmitteln der For- 
schungsvereinigung Antriebstechnik e. V. (FVA). Die Autoren bedanken sich für die Förderung und Unterstützung bei der FVA und den Mitgliedern des projektbegleitenden Ausschusses.

Funding Open Access funding enabled and organized by Projekt DEAL.

Interessenkonflikt T. Schneider, K. Völkel, H. Pflaum und K. Stahl geben an, dass kein Interessenkonflikt besteht.

Open Access Dieser Artikel wird unter der Creative Commons Namensnennung 4.0 International Lizenz veröffentlicht, welche die Nutzung, Vervielfältigung, Bearbeitung, Verbreitung und Wiedergabe in jeglichem Medium und Format erlaubt, sofern Sie den/die ursprünglichen Autor(en) und die Quelle ordnungsgemäß nennen, einen Link zur Creative Commons Lizenz beifügen und angeben, ob Änderungen vorgenommen wurden.

Die in diesem Artikel enthaltenen Bilder und sonstiges Drittmaterial unterliegen ebenfalls der genannten Creative Commons Lizenz, sofern sich aus der Abbildungslegende nichts anderes ergibt. Sofern das betreffende Material nicht unter der genannten Creative Commons Lizenz steht und die betreffende Handlung nicht nach gesetzlichen Vorschriften erlaubt ist, ist für die oben aufgeführten Weiterverwendungen des Materials die Einwilligung des jeweiligen Rechteinhabers einzuholen.

Weitere Details zur Lizenz entnehmen Sie bitte der Lizenzinformation auf http://creativecommons.org/licenses/by/4.0/deed.de.

\section{Literatur}

1. Anderson AE, Knapp RA (1990) Hot spotting in automotive friction systems. Wear 135:319-337. https://doi.org/10.1016/00431648(90)90034-8

2. Bryant D, Fieldhouse JD, Talbot CJ (2011) Brake judder-An investigation of the thermo-elastic and thermo-plastic effects during braking. Int J Veh Struct Syst. https://doi.org/10.4273/ijvss.3.1.07

3. Schneider T, Bedrikow AB, Völkel K et al (2021) Comparison of various wet-running multi-plate clutches with paper friction lining with regard to spontaneous damage behavior. Tribol Ind 43:40-56. https://doi.org/10.24874/ti.992.10.20.01

4. Hensel M, Pflaum H, Stahl K (2014) Lebensdauer Lamellenkupplungen - Abschlussbericht: Einflüsse neuartiger Reibbeläge und Öle sowie der Betriebsart auf die Lebensdauer von Lamellenkupplungen. Forschungsvereinigung Antriebstechnik e.V., Frankfurt a.M. (FVA-Nr. 515 II - Heft 1089)

5. Kasem H, Brunel JF, Dufrénoy P et al (2011) Thermal levels and subsurface damage induced by the occurrence of hot spots during high-energy braking. Wear 270:355-364. https://doi.org/10.1016/j. wear.2010.11.007

6. Schneider T, Strebel M, Pflaum H et al (2019) Spontanschädigungsverhalten von nasslaufenden Lamellenkupplungen mit organischen und metallischen Reibbelägen. Forsch Ingenieurwes 83:199-207. https://doi.org/10.1007/s10010-019-00304-1

7. Fairbank D, Maruo K, Du S et al (2001) ATF additive effects on hot spot formation in wet clutches. In: International Fall Fuels and Lubricants Meeting and Exposition; San Antonio, TX; United States; 24 September. SAE technical paper series. https://doi.org/ 10.4271/2001-01-3594

8. Hämmerl B (1995) Lebensdauer- und Temperaturverhalten ölgekühlter Lamellenkupplungen bei Lastkollektivbeanspruchung. Dissertation, Technische Universität München

9. Hirano T, Maruo K, Gu X et al (2007) Development of friction material and quantitative analysis for hot spot phenomenon in wet clutch system. SAE technical paper series. https://doi.org/10.4271/ 2007-01-0242
10. Pfleger F (1998) Schalt- und Lebensdauerverhalten von Lamellenkupplungen. Dissertation, Technische Universität München

11. Groetsch D, Stockinger U, Schneider T et al (2021) Experimental investigations of spontaneous damage to wet multi-plate clutches with carbon friction linings. Forsch Ingenieurwes 117:2480. https:// doi.org/10.1007/s10010-021-00492-9

12. Fec MC, Sehitoglu H (1985) Thermal-mechanical damage in railroad wheels due to hot spotting. Wear 102:31-42. https://doi.org/ 10.1016/0043-1648(85)90089-4

13. Fieldhouse JD, Bryant D, Talbot CJ (2011) Hot judder-An investigation of the thermo-elastic and thermo-plastic effects during braking. SAE Int J Passeng Cars Mech Syst 4:1086-1101. https://doi. org/10.4271/2011-01-1575

14. Degallaix G, Dufrénoy P, Wong J et al (2007) Failure mechanisms of TGV brake discs. Mech Behav Mater 345-346:697-700. https:// doi.org/10.4028/0-87849-440-5.697

15. Antoni G, Désoyer T, Lebon F (2012) A combined thermo-mechanical model for tribological surface transformations. Mech Mater 49:92-99. https://doi.org/10.1016/j.mechmat.2011.12.005

16. Lee K, Brooks FW (2003) Hot spotting and judder phenomena in aluminum drum brakes. J Tribol 125:44-51. https://doi.org/10. $1115 / 1.1506315$

17. Kao TK, Richmond JW, Douarre A (2000) Brake disc hot spotting and thermal judder: an experimental and finite element study. IJVD 23:276. https://doi.org/10.1504/ijvd.2000.001896

18. Yu L, Ma B, Chen M et al (2019) Investigation on the failure mechanism and safety mechanical-thermal boundary of a multidisc clutch. Eng Fail Anal 103:319-334. https://doi.org/10.1016/j. engfailanal.2019.04.071

19. Dufrénoy P, Bodovillé G, Degallaix G (2002) Damage mechanisms and thermomechanical loading of brake discs. Temp Fatigue Interact 29:167-176. https://doi.org/10.1016/S1566-1369(02)80073-5

20. Yang Z, Han J, Li W et al (2013) Analyzing the mechanisms of fatigue crack initiation and propagation in CRH EMU brake discs. Eng Fail Anal 34:121-128. https://doi.org/10.1016/j.engfailanal. 2013.07.004

21. Tang J, Bryant D, Qi H et al (2018) Simplified three-dimensional finite element hot-spotting modelling of a pin-mounted vented brake disc: an investigation of hot-spotting determinants. Proc Inst Mech Eng Part D J Automob Eng 232:877-895. https://doi.org/10.1177/ 0954407017713080

22. Barber JR (1969) Thermoelastic instabilities in the sliding of comforming solids. Proc R Soc A Math Phys Eng Sci 312:381-394. https://doi.org/10.1098/rspa.1969.0165

23. Zhao J-X, Ma B, Li H-Y et al (2015) Thermoelastic stability of wet clutches during engaging process. Jilin Daxue Xuebao (Gongxueban) 45:22-28. https://doi.org/10.13229/j.cnki.jdxbgxb201501004

24. Zagrodzki P, Truncone SA (2003) Generation of hot spots in a wet multidisk clutch during short-term engagement. Wear 254:474-491. https://doi.org/10.1016/S0043-1648(03)00019-X

25. Kubota M, Suenaga T, Kazuhiro D (1998) A study of the mechanism causing high-speed brake judder. SAE Technical Paper Series. https://doi.org/10.4271/980594

26. Kumar M, Boidin X, Desplanques Y et al (2011) Influence of various metallic fillers in friction materials on hot-spot appearance during stop braking. Wear 270:371-381. https://doi.org/10.1016/j. wear.2010.11.009

27. Zhao W, Zagrodzki P (2001) Study of wet friction material test under severe thermal and mechanical loading ("bump test"). Wear 123:224. https://doi.org/10.1115/1.1331056

28. Schneider T, Voelkel K, Pflaum H et al (2020) Friction behavior of pre-damaged wet-running multi-plate clutches in an endurance test. Lubricants 8:68. https://doi.org/10.3390/lubricants8070068

29. Acuner R, Pflaum H, Stahl K (2016) Tribologische Schädigungsmechanismen von Synchronisierungen mit Carbon-Reibwerkstof- 
fen. In: FVA (Hrsg) GETLUB - Tribologie- und Schmierstoffkongress, S 239-251

30. Stockinger U, Schneider T, Pflaum H et al (2020) Single vs. multicone synchronizers with carbon friction lining-A comparison of load limits and deterioration behavior. Forsch Ingenieurwes 214:55. https://doi.org/10.1007/s10010-020-00406-1

31. Matsumoto T (1994) The influence of paper-based friction material porosity on the performance of a wet clutch. SAE Technical Paper, Bd. 941032. https://doi.org/10.4271/941032

32. Nyman P, Mäki R, Olsson R et al (2006) Influence of surface topography on friction characteristics in wet clutch applications. Wear 261:46-52. https://doi.org/10.1016/j.wear.2005.09.020

33. Wimmer T, Pflaum H, Stahl K (2005) Lastkollektive Lamellenkupplungen: Untersuchung der Einflüsse der Lastfolge auf das Betriebsverhalten von nasslaufenden Lamellenkupplungen. Forschungsvereinigung Antriebstechnik e. V., Frankfurt a.M. (FVA Nr. 413 I - Heft 754)

34. Li M, Khonsari MM, McCarthy DMC et al (2015) On the wear prediction of the paper-based friction material in a wet clutch. Wear 334-335:56-66. https://doi.org/10.1016/j.wear.2015.04.005

35. Hensel M (2014) Thermische Beanspruchbarkeit und Lebensdauerverhalten von nasslaufenden Lamellenkupplungen. Dissertation, Technische Universität München

36. Meingaßner GJ, Pflaum H, Stahl K (2015) Test-rig based evaluation of performance data of wet disk clutches. In: 14th International CTI Symposium
37. Voelkel K (2020) Einflüsse des Reibsystems auf das Einlaufverhalten von Lamellenkupplungen: Abschlussbericht. Forschungsvorhaben, Bd. 343 IV. Forschungsvereinigung Antriebstechnik e. V., Frankfurt

38. Voelkel K, Pflaum H, Stahl K (2016) KUPSIM 3.0 Abschlussbericht: Erweiterung der Simulationsmethodik zur Berechnung des instationären Temperaturverhaltens nasslaufender Lamellenkupplungen. Forschungsvereinigung Antriebstechnik e. V., Frankfurt a.M. (FVA-Nr. 150/VII - Heft 1204)

39. Strebel M, Pflaum H, Stahl K (2016) Einflüsse auf die Spontanschädigung von nasslaufenden Lamellenkupplungen mit organischen Reibbelägen. In: FVA (Hrsg) GETLUB - Tribologie- und Schmierstoffkongress, S 99-110

40. Strebel M (2017) Spontanschäden an nasslaufenden Lamellenkupplungen. Dissertation, Technische Universität München

41. Meingaßner GJ (2017) Methodik zur Untersuchung des Reibungsverhaltens nasslaufender Lamellenkupplungen bei Langsamlaufund Mikroschlupf. Dissertation, Technische Universität München

42. Duminy J, Federn K (1977) Ermittlung der Leistungsgrenzen der Reibpaarung Stahl/Sinterbronze, in ölgeschmierten Reibungskupplungen unter Berücksichtigung des Einflusses der Kühlund Schmiermittel. Forschungsvereinigung Antriebstechnik e. V., Frankfurt a.M. (FVA Nr. 9 I - Heft 52)

43. Acuner R (2016) Synchronisierungen mit Carbon-Reibwerkstoffen unter hohen und extremen Beanspruchungen. Dissertation, Technische Universität München 